\title{
T Tauri Disk Lifetime in the Lupus Association
}

\author{
P. A. B. Galli ${ }^{1}$, C. Bertout ${ }^{2}$, R. Teixeira ${ }^{1}$ and C. Ducourant ${ }^{3,4}$ \\ ${ }^{1}$ Instituto de Astronomia, Geofísica e Ciências Atmosféricas, Universidade de São Paulo, \\ Rua do Matão, 1226 - Cidade Universitária, 05508-900, São Paulo - SP, Brazil. \\ email: galli@astro.iag.usp.br \\ ${ }^{2}$ Institut d'Astrophysique, 98bis, Bd. Arago, 75014 Paris, France. \\ ${ }^{3}$ Univ. Bordeaux, LAB, UMR 5804, F-33270, Floirac, France. \\ ${ }^{4}$ Observatoire Aquitain des Sciences de l'Univers, CNRS-UMR 5804, 33270 Floirac, France.
}

\begin{abstract}
In a recent study, we derived individual distances for a sample of pre-main sequence stars that define the comoving association of young stars in the Lupus star-forming region. Here, we use these new distances to investigate the mass and age distributions of Lupus T Tauri stars and derive the average disk lifetime in the Lupus association based on an empirical disk model.
\end{abstract}

Keywords. stars: variables: T Tauri, Herbig Ae/Be - stars: formation - stars: evolution

\section{Introduction}

It has been hypothesized for a long a time (Bertout 1989) that classical T Tauri stars (CTTSs), which are actively accreting from circumstellar disks, become weak-line T Tauri stars (WTTSs), which show no spectroscopic evidence for accretion, when their disks dissipate. This implies that the timescale of disk dissipation should be only a few Myr to explain both subgroups coexisting in the Hertzsprung-Russell diagram (HRD). Bertout et al. (2007) obtained for the first time the average lifetime of a circumstellar disk in terms of the mass of the parent star in the Taurus-Auriga star-forming region. However, the evolution of circumstellar disks is expected to depend on environmental criteria. Here, we investigate whether the different environment of the Lupus star-forming region affects the lifetime of the circumstellar disks in this region.

\section{Physical properties of the Lupus comoving stars}

The sample of stars used in thus study is based on the comoving stars of the Lupus association with individual distances derived by Galli et al. (2013). Using the photometric and spectroscopic information available in the literature we compute the photospheric luminosity of 92 T Tauri stars (TTSs), and estimate their masses and ages from the Siess et al. (2000) evolutionary models. Fig. 1 illustrates the HRD of the Lupus association.

We investigated the mass and age distribution of the CTTSs and WTTSs in Lupus and concluded from KS-tests and Wilcoxon rank sum tests that the probability of both TTS subclasses being drawn from the same age distribution is very low. The CTTSs are on average younger than WTTSs and their distribution in the HRD (see Fig. 1) is shifted towards lower masses and late spectral types. We also confirmed in this work that the more dispersed "off-cloud" WTTSs surrounding the Lupus clouds are older than the "on-cloud" population of WTTSs located in the direction of the main star-forming clouds of the complex (Lupus 1-4). 


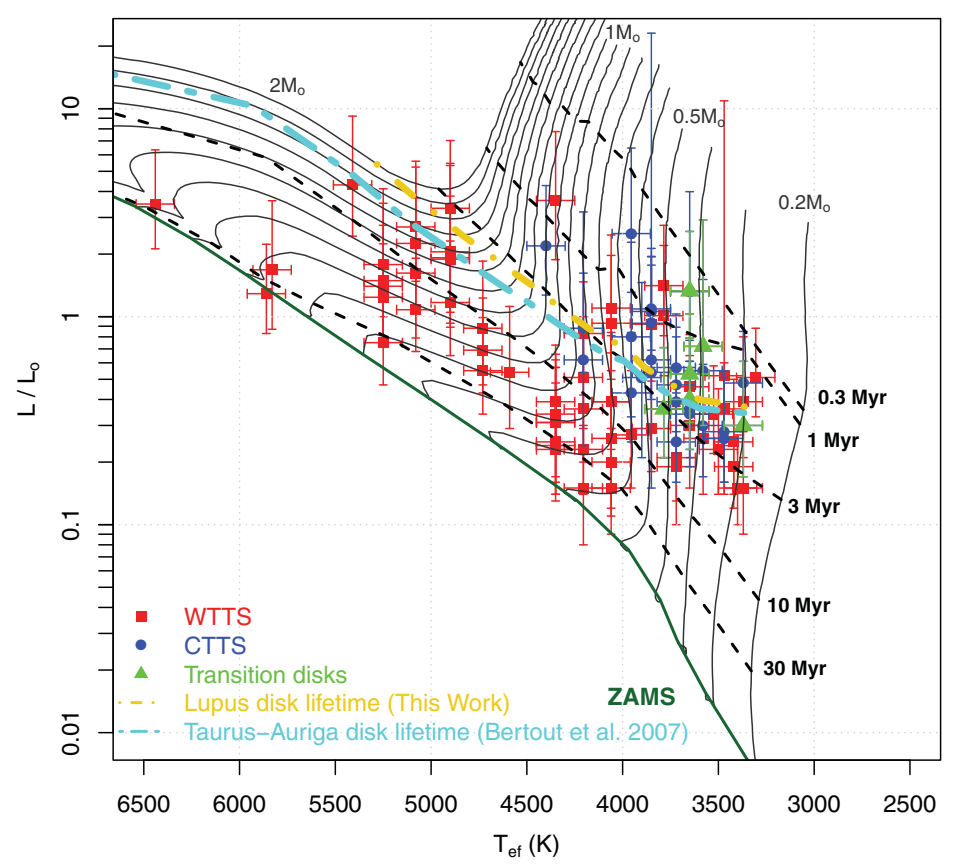

Figure 1. HRD of the Lupus association using the Siess et al. (2000) models.

\section{The evolution from CTTS to WTTS}

Following the disk model developed by Bertout et al. (2007), we assume that the disk mass of a TTS is given by $M_{d}=\alpha\left(M / M_{\odot}\right)^{\beta}$ while the mass accretion rate (in units of $\left.M_{\odot} / y r\right)$ is given by $\dot{M}_{a c c}=\gamma\left(M_{\star} / M_{\odot}\right)^{2.1}$. The disk lifetime $\tau_{d}=M_{d} / \dot{M}_{a c c}$ can be written as $\log \tau_{d}=\log (\alpha / \gamma)+(\beta-2.1) \log \left(M_{\star} / M_{\odot}\right)$, where $\alpha, \beta$ and $\gamma$ are parameters to be determined from the modeling, using the stellar masses derived in this work.

The model predicts that the star is a CTTS, if $t_{\star} \leqslant \tau_{d}\left(M_{\star}\right)$, and a WTTS, if $t_{\star}>$ $\tau_{d}\left(M_{\star}\right)$. We compare the mass distribution of the modeled CTTSs (and WTTSs) with the observed CTTSs (and WTTSs) in our sample, and run a KS-test to find the best match between these populations for a given combination of $\alpha, \beta$ and $\gamma$. We vary these parameters in the range of $5 \leqslant \log (\alpha / \gamma) \leqslant 8$ and $-4 \leqslant \beta \leqslant 4$ in steps of 0.01 . Doing so, we find $\log (\alpha / \gamma)=6.53 \pm 0.05$ and $\beta=2.65 \pm 0.10$. This implies that the average disk lifetime in the Lupus association predicted by our model is $\tau_{d} \simeq 3 \times 10^{6}\left(M_{\star} / M_{\odot}\right)^{0.55} \mathrm{yr}$.

The average disk lifetime given by this model for the Taurus-Auriga association (see Bertout et al. 2007) is $\tau_{d} \simeq 4 \times 10^{6}\left(M_{\star} / M \odot\right)^{0.75}$ yr. Thus, our heuristic model predicts that the two associations may have different disk lifetime mass dependencies. Although this result will need confirmation with statistically more significant samples, it gives a first indication that disk lifetimes can be different in different star-forming regions.

\section{References}

Bertout, C. 1989, ARA\&A, 27, 351

Bertout, C., Siess, L., \& Cabrit, S. 2007, A\&A, 473, L21

Galli, P. A. B., Bertout, C., Teixeira, R., \& Ducourant, C. 2013, A $6 A$, 558, A77

Siess, L., Dufour, E., \& Forestini, M. 2000, AछA, 358, 593 